\section{Case Reports in Ophthalmology}

Case Rep Ophthalmol 2016;7:354-358

DOI: $10.1159 / 000447995$ Publisnea oninne: Juny ZI, 2016
(C) 2016 The Author(s)

Published by S. Karger AG, Basel www.karger.com/cop

This article is licensed under the Creative Commons Attribution-NonCommercial 4.0 International License (CC BY-NC) (http://www.karger.com/Services/OpenAccessLicense). Usage and distribution for commercial purposes requires written permission.

\title{
Swept-Source Optical Coherence Tomography Detecting Intraoperative Acute Descemet's Fold Formation
}

\author{
Yu Ichioka Akihito Uji Nagahisa Yoshimura \\ Department of Ophthalmology and Visual Sciences, Kyoto University Graduate School of \\ Medicine, Kyoto, Japan
}

\section{Keywords}

Optical coherence tomography · Swept-source optical coherence tomography .

Descemet's membrane $\cdot$ Descemet's fold

\begin{abstract}
Background: To present an intraoperative acute Descemet's fold formation using sweptsource optical coherence tomography (SS-OCT) imaging. Case Report: A 67-year-old man complaining of reduced visual acuity in the left eye. A 25-gauge pars plana vitrectomy combined with phacoemulsification cataract surgery was performed to remove the vitreomacular traction. When hydro-sealing was performed, striae rapidly spread in the cornea. SS-OCT Bscan images performed on postoperative day 1 revealed a wavy Descemet's membrane that might correspond to Descemet's folds. Pairs of hypo- and hyperreflective narrow lesions running from the wavy Descemet's membrane to almost half of the thickness of the whole cornea were observed. En face OCT imaging clearly showed the stromal fold, which continuously spread from the Descemet's fold. Conclusion: The stromal fold might be due to the focal bulge of the stroma posteriorly caused by the rapid volume increase of the stroma which could push Descemet's membrane posteriorly, thereby forming a wavy Descemet's membrane layer.

(C) 2016 The Author(s) Published by S. Karger AG, Basel
\end{abstract}




\section{Case Reports in Ophthalmology}

\section{Introduction}

Edematous thickening of the corneal stroma due to endothelial dysfunction can shift Descemet's membrane posteriorly and cause a surplus of Descemet's membrane, also known as Descemet's fold. Postoperative transient corneal enema and Descemet's fold are major complications of phacoemulsification surgery; they usually appear during the early postoperative period and rarely occur intraoperatively $[1,2]$. Here, we present an intraoperative occurrence of Descemet's fold which spread rapidly during corneal hydration in phacoemulsification surgery and was documented using swept-source optical coherence tomography (SS-OCT) imaging for the first time.

\section{Case Report}

A 67-year-old man complaining of reduced visual acuity in the left eye was referred to our institution. He had a history of diabetes mellitus. His visual acuity was 20/40 in the left eye, and panretinal photocoagulation had been performed for diabetic retinopathy in both eyes. He had a mild nuclear cataract (grade 1 Emery-Little classification), but no other abnormal findings were found in the anterior segment. Fundus examination revealed cystoid macular edema in the left eye with incomplete posterior vitreous detachment. Spectral domain OCT showed a thickened posterior vitreous membrane and obvious macular traction.

A 25-gauge pars plana vitrectomy combined with phacoemulsification cataract surgery was performed to remove the macular traction. After sub-Tenon's anesthesia of $2 \%$ xylocaine, two corneal side ports (located at 9 and 2 o'clock) and a 2.4-mm transconjunctival sclerocorneal incision (10 o'clock) were made. A continuous curvilinear capsulorhexis of 5.5 $\mathrm{mm}$ was completed via an ophthalmic viscosurgical device (OPEGAN Hi ${ }^{\circledR}$; Seikagaku Corporation, Tokyo, Japan), followed by hydrodelineation and hydrodissection. Phacoemulsification and aspiration were performed using a CONSTELLATION ${ }^{\circledR}$ Vision System (Alcon Laboratories, Inc., Fort Worth, Tex., USA), and a three-piece hydrophobic acrylic intraocular lens (Avansee; Kowa, Tokyo, Japan) was implanted in the capsular bag. When hydro-sealing was performed at the temporal side of the corneal incision with a balanced salt solution, striae rapidly spread in the cornea. The faint Descemet's fold with hazy corneal stroma was formed (fig. 1). Although the change did not diminish until the end of the surgery, visibility of the fundus was sufficient to accomplish vitrectomy with inner limiting membrane peeling.

On postoperative day 1, Descemet's fold and stromal edema were still observed. SS-OCT (DRI OCT-1; Topcon, Tokyo, Japan) B-scan images visualize the center of the cornea and reveal a wavy Descemet's membrane and an endothelium layer that might correspond to Descemet's folds (fig. 2a, b). These layers did not seem thickened. Moreover, pairs of hypoand hyper-reflective narrow lesions running from the wavy Descemet's membrane to almost half of the thickness of the whole cornea were observed. En face OCT images were generated by using built-in software (Enview; Topcon, Tokyo, Japan) to investigate the threedimensional structure of these findings [3]. To obtain a stack of images parallel to the corneal surface, the flattening function, which was originally developed for automatic correction of the concavity of the posterior pole, was employed. In this study, flattening was performed at the level of the anterior corneal surface, which was mistaken for the inner limiting membrane by the software. En face OCT images displayed a white grid, which corresponded to the wavy folds at the level of Descemet's membrane (fig. 2e). This is considered identical to Descemet's fold. Intriguingly, the same grid formation was detected in the corneal stroma, 
Case Reports in
Ophthalmology

Case Rep Ophthalmol 2016;7:354-358 $10.1159 / 000447995$ www.karger.com/cop

Ichioka et al.: Swept-Source Optical Coherence Tomography Detecting Intraoperative Acute Descemet's Fold Formation

which corresponded to hyporeflective narrow lesions in the B-scans (fig. 2d). These grids could be detected in the en face OCT images from the level of Descemet's membrane to half of the thickness of the whole cornea (fig. 2c) but not in the anterior half of the cornea. On postoperative day 36 , these findings were detectable, but not remarkable.

\section{Discussion}

The mechanism of the acute Descemet's fold formation reported here might be the secondary change due to acute swelling of the corneal stroma. Direct irrigation of the balanced salt solution beneath Descemet's membrane might be an alternative explanation. Considering the lack of Descemet's membrane detachment and the presence of the acute stromal changes, it is reasonable to speculate that the acute stromal thickening caused by hydration was the origin.

En face OCT imaging clearly presented the stromal fold, which continuously spread from the Descemet's fold. The stromal fold might be due to the focal bulge of the stroma posteriorly caused by the rapid volume increase of the stroma which could push Descemet's membrane posteriorly, thereby forming a wavy Descemet's membrane layer. The reason why the bulge of the stroma was formed in the posterior part of the stroma might be due to the histologic difference in lamellas between the anterior and posterior stroma [4]. Namely, greater swelling occurs more easily in the posterior corneal stroma, which is composed of parallel collagenous lamellas; whereas the anterior corneal stroma is composed of obliquely arranged lamellas.

In conclusion, the stromal and Descemet's fold might be due to the focal bulge of the stroma posteriorly induced by the rapid volume increase of the stroma and can be caused by hydration independently of endothelial dysfunction.

\section{Statement of Ethics}

This study adhered to the tenets of the Declaration of Helsinki and was approved by the Institutional Review Board at Kyoto University Graduate School of Medicine for retrospective review of existing patients' data.

\section{Disclosure Statement}

The authors have no conflicts of interest to disclose.

\section{References}

1 Do JR, Oh JH, Chuck RS, Park CY: Transient corneal edema is a predictive factor for pseudophakic cystoid macular edema after uncomplicated cataract surgery. Korean J Ophthalmol 2015;29:14-22.

-2 Tao A, Chen Z, Shao Y, Wang J, Zhao Y, Lu P, Lu F: Phacoemulsification induced transient swelling of corneal Descemet's endothelium complex imaged with ultra-high resolution optical coherence tomography. PLoS One 2013;8:e80986.

-3 Alasil T, Ferrara D, Adhi M, Brewer E, Kraus MF, Baumal CR, Hornegger J, Fujimoto JG, Witkin AJ, Reichel E, Duker JS, Waheed NK: En face imaging of the choroid in polypoidal choroidal vasculopathy using swept-source optical coherence tomography. Am J Ophthalmol 2015;159:634-643.

4 Fine BS, Yanoff M: Ocular histology: a text and atlas. New York, Medical Dept., Harper and Row, 1972. 
Case Reports in
Ophthalmology

Case Rep Ophthalmol 2016;7:354-358

(C) 2016 The Author(s). Published by S. Karger AG, Basel www.karger.com/cop

Ichioka et al.: Swept-Source Optical Coherence Tomography Detecting Intraoperative Acute Descemet's Fold Formation
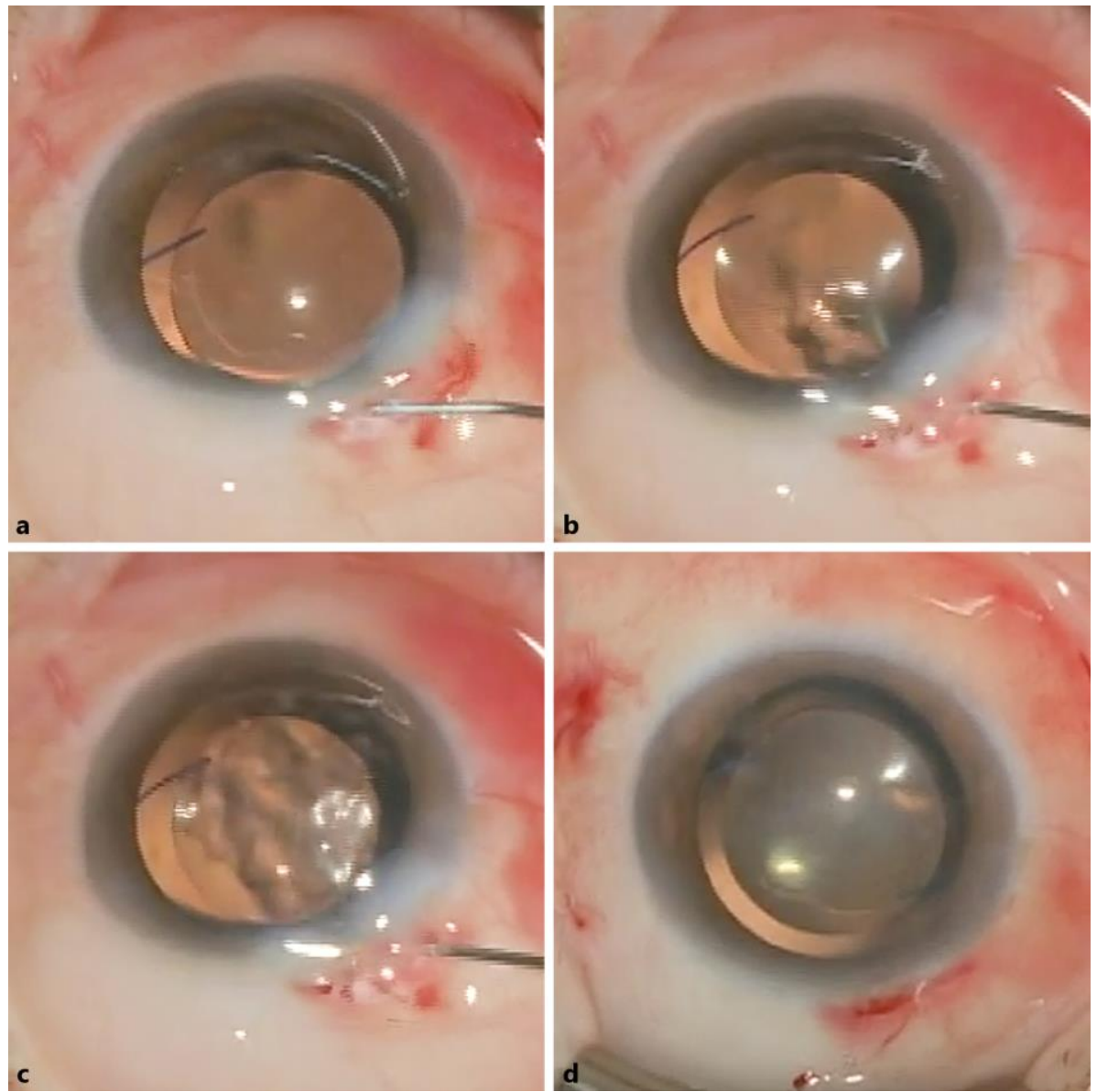

Fig. 1. Striae rapidly spread in the cornea during hydro-sealing. a Before hydration at the temporal side of the corneal incision. b, c Imaging of striae spread during hydro-sealing. $\mathbf{d}$ At the end of surgery a faint Descemet's fold with hazy corneal stroma is formed. 


\section{Case Reports in Ophthalmology}
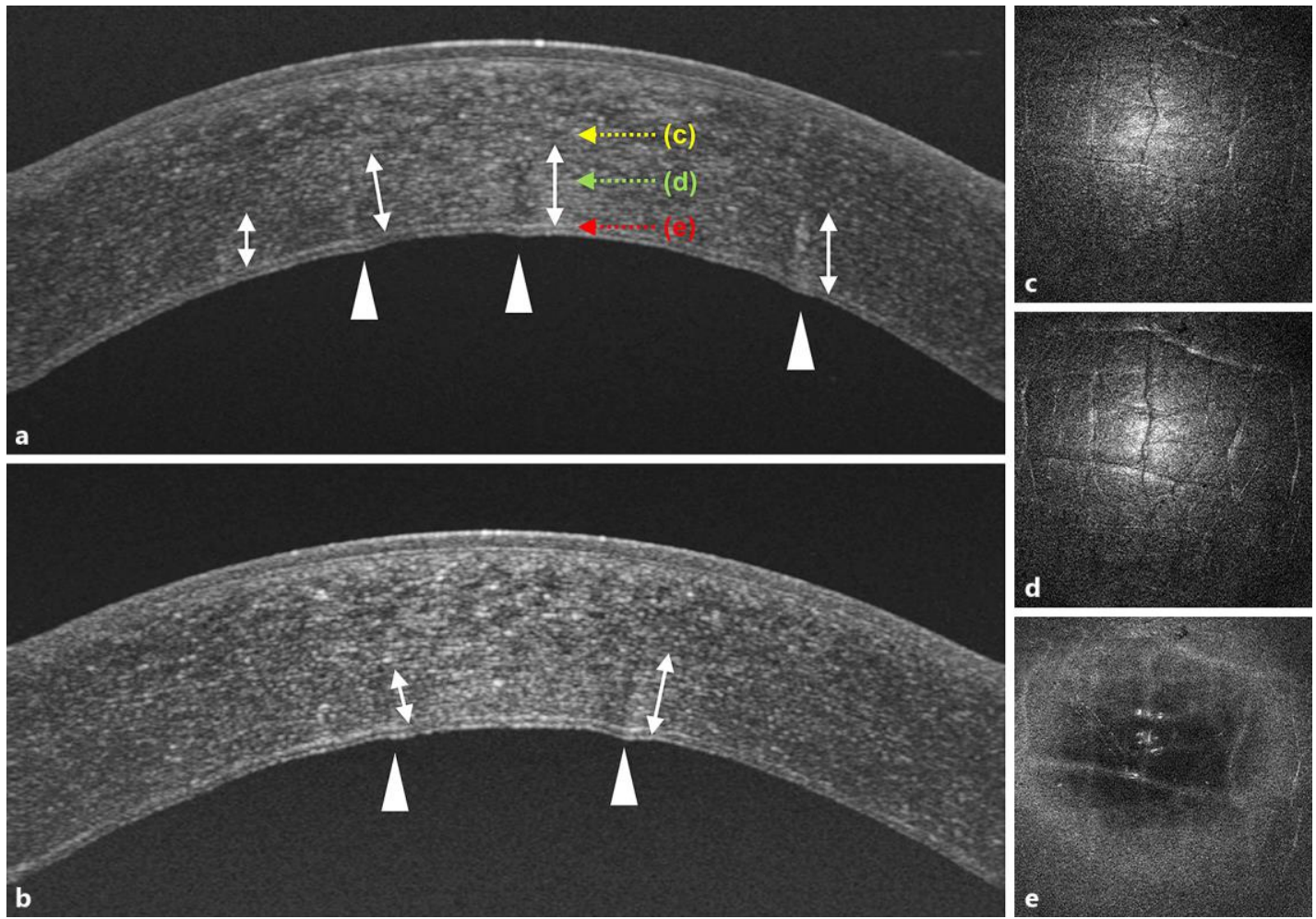

Fig. 2. SS-OCT imaging of the cornea performed on postoperative day 1. a Horizontal SS-OCT B-scan image shows wavy Descemet's membrane and endothelium layer (arrowheads) and the pairs of hyporeflective and hyperreflective narrow lesions (double-headed arrows). b Vertical SS-OCT B-scan image. c-e En face OCT images showing grid-patterned stromal fold, which continuously spreads from Descemet's fold. c En face OCT image at the level of half of the thickness of the whole cornea corresponding to the depth indicated by the yellow arrow in panel a. $\mathbf{d}$ En face OCT image at the level indicated by the green arrow in panel a. e En face OCT image at the level indicated by the red arrow in panel a. 